\title{
CAN ADDITIVE MANUFACTURING HELP WIN THE RACE?
}

\author{
Clive Henry Hands ${ }^{1}$, Anton du Plessis ${ }^{2}$, Nicholas Minnaar ${ }^{3}$, Byron Alexander Blakey- \\ Milner $^{1}$, Ernst Burger ${ }^{3}$
}

\begin{abstract}
This paper provides an overview of the new CPAM Project on Additive Manufacturing (AM) in design and simulation, focusing on topology \& lattice structure optimization for a lightweighting advantage. This industry/academia collaboration project aims to utilize existing hardware and software tools, and investigate the practical limits of the technologies, providing eventual guidelines for general use. This will provide a solid foundation for the practical use of metal AM optimized solid and latticed structures especially for Ti6Al4V parts. Two case studies are demonstrated here, one a purely topology optimized design, and one also incorporating lattice optimized design, both from Ti6Al4V and load-bearing components, to be utilized in the Nelson Mandela University (NMU) Eco-Car Project in competition, late in 2018. This paper presents the Design for Additive Manufacturing (DfAM) process, the challenges met iro applying a DfAM design mindset, and a unique final voxel-based smoothing step finishing off the design process. Detailed structural integrity assessment of these parts are included - the question remains: can Additive Manufacturing help win the race?
\end{abstract}

\section{INTRODUCTION}

The newly established CPAM project in lattice structure designs has provided an opportunity to investigate the practical limits and challenges of this technology, using state of the art hardware and software tools. The project is aimed at investigating and advancing the use of lightweight and lattice designs in $A M$, from fundamental aspects to practical application.

While the topic is widely known and reviewed in more detail in (Yanget al[1]), its practical use is actually limited by the wide design space: the user is not normally sure what design constraints to use, where in the part to use lattices or what kind of lattice designs are possible to be practically incorporated. Currently the designs created are limited by typical settings in the software packages used, and not yet fully exploiting the complexity available. Furthermore, the manufacturability and suitability of different lattice designs are still in their infancy. Current work in progress in this project is aimed at analyzing different lattice unit cell designs, investigating manufacturability, directional mechanical properties, permeability and other parameters which vary for different lattice designs (du Plessis et al[2]).

Current state of the art lattice structure production by $\mathrm{AM}$ has been reviewed recently for bone replacement implants (Zhang et al[3]; Wang et al[4]). Other light-weighting applications using lattices are widely studied but few examples exist in literature thus far; a good example is reported in (Orme et al[5]). Besides lattice designs, manufacturing limits exist which affect mechanical properties of lattices and parts containing lattices. This project will further investigate this: particularly internal porosity in struts of lattices, directionally-dependent thickness variations in production (eg. thicker horizontal struts vs vertical struts), and surface roughness all play a role in strength, stiffness and fatigue life of lattice parts and need further investigation to provide suitable guidelines for latticed designs.

\footnotetext{
${ }^{1}$ Department of Mechanical Engineering, Nelson Mandela University, Port Elizabeth, South Africa

${ }^{2}$ CT Scanner Facility, University of Stellenbosch, South Africa

${ }^{3}$ Altair SA, Stellenbosch, South Africa
}

Presented at the 19th Annual International RAPDASA conference, held from 7 to 9 November 2018 in Johannesburg, South Africa. 
Finally, to fully exploit the complex design space, it's possible to incorporate biomimetic design rules, ie. using design concepts from nature. This is further investigated by using specific biological structures and analyzing their successful structural details in terms of lattice and lightweight design - one recent investigation is reported for impact protective design based on design of an animal's body armour (du Plessis et al[6]), and its application to metal additively manufactured body armour units, reported in (du Plessis et al[7]).

MicroCT use is crucial in quality inspection of produced parts, as has been reviewed in (du Plessis et al[8]). This kind of inspection and analysis becomes especially important for thinwalled components and latticed parts which may contain internal porosity or unconsolidated powder, or may have small cracks from unexpected residual stresses, due to the complex shapes produced. By combining all above topics, this project aims towards practical application, with load bearing parts designed using incorporated topology- and lattice optimization. The hope is that this project will deliver practical guidelines assisting users in the wider adoption of the methods of topology optimized and latticed AM parts.

Topology Optimized design for $A M$ is a topic of intense research and application currently, resulting in efficient design by expanding available design space and allowing greater design complexity as a result of design freedom offered by additive manufacturing (Liu et al[9]).

Its main purpose is to produce an efficient design by either light-weighting the part or maximizing the stiffness; this is achieved by using pre-defined inputs (loads, constraints, materials, joints \& contacts) via simulation to determine which areas of the design space require material as a result of the load paths, while not affecting the strength or deformation of the structure. This process is then iterated to result in an optimized structure.

As a next step, it is possible to add lattice structures to the optimized design space where low stresses are found, or where stiffness can be tailored to suit the application. The output structure often requires some data post-processing in the form of smoothing and defining solid support material regions, to deliver a practical design input for additive manufacturing. All of this is typically done in Topology Optimization enabled software such as in Altair Inspire, in tandem with specialized AM preparation software, all of which was used in this work.

The former software, which incorporates many state-of-the-art technology features that will be further detailed in Section 3, facilitates simulation-driven design exploration and its use in the design process. Applications similar to ones documented in this project have been validated many times over as evidenced by a number of commercial success stories from vendors (eg. Robot Bike Company[10], Ryerson University[11] \& Gator Motorsports[12]).

\section{THE ECO-CAR PROJECT}

The NMU Eco-Car Project was initiated off the back of the institution's Solar Car Project back in 2014 when that project became too expensive to continue. With the focus on design for light-weighting, in tandem with the carbon compositing experience already established via the Solar Car project, the Eco-Car Project was deemed an ideal fit to continue with the research done into lightweighting and specifically Topology Optimization techniques.

The establishment of the annual Shell Eco-Marathon: South Africa in 2014 provided an ideal outlet for this project. The Shell Eco-Marathon (SEM) is a global event held in many countries with the Americas, European and Asian events considered the main blue-flag events internationally, with several newer events like South Africa being termed Challenger events.

The intent of the SEM is for student teams to conceive, design, manufacture and compete in the event to try and achieve the furthest possible distance on an equivalent 1 litre of standard fuel. It is a multi-disciplinary project directly tying in with the current global engineering strategies being prioritized by OEMs of light-weighting, efficiency, fuel-saving, advanced design and manufacturing techniques applied to the automotive and aerospace supply chain. 
Two main categories exist in the SEM: Prototype and Urban Concept, the South African event catering only for Prototype category. There are also several sub-classes iro power source, with the local event only allowing Electrical and Internal Combustion Engine (ICE) options.

The NMU Eco-Car (Figure 1) chose to use ICE as their power option mainly due to the established prevalence of automotive manufacturers in the Eastern Cape region using traditional ICE options, but are considering an Electric option in the future - this was preconceived in the initial modular design to allow for easy swapping of power sources.

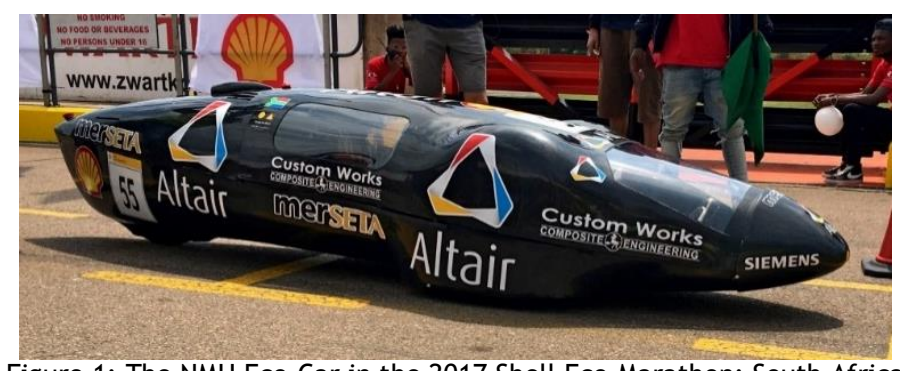

Figure 1: The NMU Eco-Car in the 2017 Shell Eco-Marathon: South Africa

The NMU Eco-Car Team has been fortunate to be Champions in their category in the last two events, 2016 \& 2017, setting consecutive event records on each occasion and are hoping to achieve an even better distance with the latest design iteration sporting several metal AM components in a continuous effort to lighten the car and to optimize the design even further.

In this paper, two case studies will be detailed, consisting of:

i. a topology optimized mounting C-bracket, and

ii. a topology optimized and latticed large steering arch.

\subsection{Project Initiation}

The project initiated through Altair SA's Ernst Burger establishing contact between the University of Stellenbosch's Prof Anton du Plessis and the NMU Eco-Car Team, as a result of the common bond of use of Altair software products, and the shared interest in developing Metal AM techniques, Topology Optimization \& Lattice Design for Light-weighting purposes.

Prof du Plessis had sourced funding supported by the South African Collaborative Program in Additive Manufacturing (CPAM) to conduct research into lattice structure design and application. Additionally, his current research efforts involve the standardization of MicroCT non-destructive analysis techniques to improve accessibility and cost effectiveness of the process, in order to promote wider uptake of the process to users in the AM community.

As such, an applied project was needed to test designed \& optimized metal AM components in a real-world application, which the Eco-Car Project provided, and so proved a perfect fit for all to achieve their objectives. The combined Project was thus established in mid-Feb 2018 and soon evolved into a multi-pronged program which continues to expand promisingly.

\subsection{Component Identification}

The Eco-Car's design was conceived on the basis of shrink-wrapping the aero package around a full carbon-fibre monocoque consisting of certain core components: the driver, wheels \& engine - after that, the design freedom was reasonably unrestricted.

The expense of the composite monocoque \& bodyshell components means focus on successive iterations has been on increasing efficiency and light-weighting of inner components rather 
than re-design - with the aim to further light-weight by $5[\mathrm{~kg}]$ from a version-1 mass of 52 $[\mathrm{kg}]$. As each partial gram is shed, this target proves an increasingly difficult challenge.

This door opened by the metal AM option allowed not only light-weighting on certain chosen components, but enabled more sophisticated designs utilizing both topology optimization and lattice-design techniques, further stiffening of components iro structural performance, as well as consolidation in the number of parts required to perform exactly the same task.

This led to a selection option iro which components were prioritized within the strictures of the funding allocation - a wish-list was created and then honed down. An initial urgent need for a round-robin test saw the front corner C-Bracket being chosen as first priority, and then the Steering Arch was selected as a primary load-bearing structure (Figure 2). The process behind the development and final printing of these components will be detailed next.
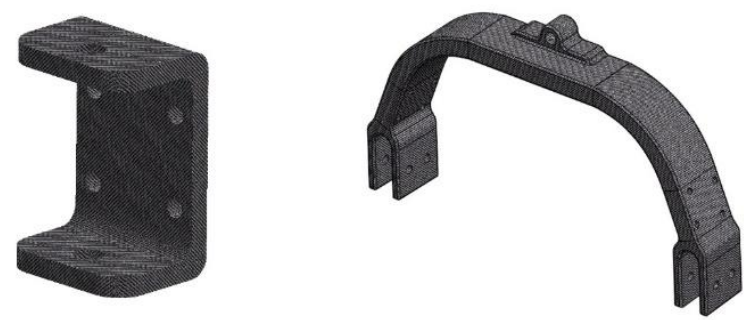

Figure 2: original C-Bracket \& Steering Arch designs

Further components are also being considered depending on funding and manufacture availability, with the hope that the completed components could be installed on the Eco-Car in time for the 2018 Shell Eco-Marathon: South Africa in October.

\section{DESIGN TECHNOLOGIES FOR ADDITIVE MANUFACTURE}

The performance of a load-bearing structure may be measured in various ways eg. max. sustainable load, mass, compliance/stiffness. Meanwhile, a factor which greatly affects these performance measures in a structure is the layout of material within its boundaries. The design of this material layout is a complex task since it presents designers with many degrees of freedom, most of which effect structural performance in non-intuitive ways.

One method for handling this design complexity is to inform the design process using Topology Optimization (TO). Additional design strategies such as replacement in the design domain of continuous material with lattice units can lead to even greater structural performances. This section will describe the technologies which yield optimal structural performances in $A M$ products, and Altair Inspire has been used in this project to administer these technologies.

\subsection{Topology Optimization}

Topology Optimization is an algorithmic approach in which structural performance iteratively drives the modification of material layout in a load-bearing structure. The algorithm has been implemented on top of finite element analysis (FEA) codes, since it is FEA which is utilized for the evaluation of structural performance. While Topology Optimization only found application in niche designs in the distant past, the technology is now well integrated into many modernized industries (eg. aerospace (Zhuet al[13]) and automotive (Yildiz[14])).

The AM industry in particular has the most to gain from Topology Optimization since a synergy exists between the two technological fields. Specifically, AM releases manufacturing freedom while TO releases design freedom in a product development process. The marriage of these two technologies therefore truly liberates the product's optimal performance as a structure.

Adoptions have previously been made to TO implementations to account for the manufacturing constraints of traditional manufacturing processes (Vatanabe et al[15]) as the optimizations inherently produce concepts with complex shapes and are thus not generally 
manufacturable (Gibson et al[16]). Until recently, however, no adoptions had been available to address a significant limitation in the space of AM. More specifically, in the case of metal laser powder bed fusion, significant support structure may be required for production since high residual stresses cause warpage. The support structure is therefore necessary to facilitate a warpage-reducing connection between the part and its print-bed base. However, support structure also drives waste production and induces the need for sometimes significant post-manufacture-processing. To mitigate these drawbacks, one strategy for minimizing the necessary support structures is to limit overhang angles in the design from the offset.

Overhang angles describe the angle between the vector which connects consecutive points on the boundaries of part and the vector parallel to the build plate (Figure 3). Post-optimization, ad-hoc modifications have typically been made to part designs to mitigate overhang angle violations from the results of Topology Optimization.

Optistruct (Optistruct User Guide[17]), which forms the kernel of Altair Inspire 2018, offers a state-of-the-art TO adoption which has been leveraged in this project to reduce overhang where practical. The technology is available via two implementations. In the first implementation, the topology of the optimized design may be strictly prohibited if it violates overhang angle constraints (Gaynor et al[18]).

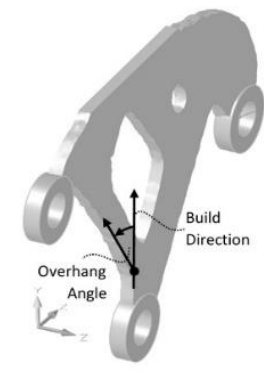

Figure 3: over-hang angle relative to build direction

Secondly, unique to Optistruct, designers may penalize overhang angles in their designs instead of removing them completely. This is achieved using a method whereby the objective of the optimization is penalized in a predefined proportion to the degree of overhang violations in the generated designs. This offers superior convergence and a mechanism to tune designs towards functionality, manufacturability, or perfect balance between the two.

\subsection{Lattice Optimization}

A state-of-the-art feature in some FEA codes, exclusive to designs that are intended for AM, is the ability to replace solid and continuous design regions with optimized lattice-unit structures. These lattice structures are efficient from multiple perspectives eg. they have high strength-to-weight ratios structurally, high surface area-to-volume ratios thermally, and they exhibit high biomedical compatibility due to their effective porosity (Helouet al [19]). In addition, the utilization of lattice-units in a design process can be coupled onto the results of a TO. Resulting designs are radically different from ones designed using traditional design strategies, an example is the steering arch designed in this project.

Individual lattice units (Figure 4) exhibit unique structural behavior since they have unique configurations whereas every unit of continuous material behaves identically. Lattice-units thus essentially allow designers to utilize localized effective material properties instead of applying a single global material property to the entire design domain.

The expense of this design freedom, however, is that the designer introduces many new design variables into the process since each unit of lattice in the domain needs to be assigned a configuration and

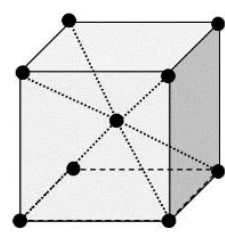

Figure 4: lattice units associated dimensions. To mitigate this multitude of design variables, designers can offload the complexity onto optimization-enabled FEA codes to generate lattice configurations and optimization technology to choose the best values for the associated dimensions.

The fundamental design variable in the Topology Optimization stage of this project, and in general, is the element-by-element material density within the design domain. Since intermediately dense material cannot be used during fabrication of a part, optimal element densities in the final optimization results of a pure TO are mapped to a discrete solution. This means that all elements in the design domain are forced to be either fully dense or void. 
The lattice optimization step offers a pragmatic solution for fabricating intermediately dense elements if coupled to the TO. This is because lattice units have an effective intermediate density when compared to a fully solid unit of equal volume. This is implemented by introducing a second optimization step where the optimal topology (or subset of the topology) from the pure TO step is replaced by lattice elements; the associated strut diameters are optimized with a constraint on the incident stress. The lattices produced are conformal to the geometry of the part and correspond to the tetrahedral mesh used for simulation. It is not currently possible to incorporate more complex lattice designs into TO designs.

\section{METHODOLOGY AND RESULTS}

\subsection{Case Study 1: C-Bracket Design Process}

\subsubsection{Initial Design Need}

The front corner C-Bracket (Figure 5) was first choice as a pilot component because:

- it was small,

- it could benefit from further stiffening,

- a lightweighting advantage could possibly be gained, and

- a solution was relatively quickly achievable.

The function of the part is to connect the front corners to the steering arch, which in turn was bonded in to the monocoque/tub of the vehicle. The performance of all current structures had already proved themselves in past events.

The original structure was a multi-layered, full carbon structure, laid-up utilizing a simple long mould structure with many layers of stacked fabric. The cured product then cut to size and each bracket subsequently post-processed via standard machining processes.

It was decided to carry out the design optimization using purely topology optimization to keep things relatively simple for the pilot project due to the urgent need, to test whether the physical outcome and envisaged outcome were comparable, and to limit the possible challenges coming out of the whole print process.

\subsubsection{Design \& Optimization Process}

Geometric properties were captured from both existing CAD documents and verified by rigorously measuring existing physical parts. A plastic $3 \mathrm{~d}$ printed $\mathrm{AM}$ test piece was then also produced to verify correct fitment into the vehicle.

The Design Space (Figure 6) of the original component was then expanded to its logical extents to maximize the volume for the topology optimization process, in order to ensure as many variants of solution as possible.

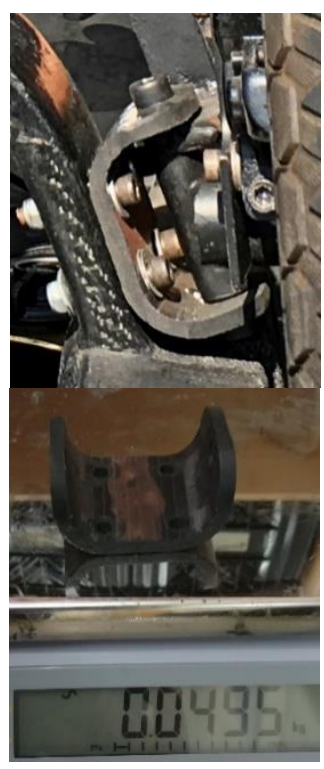

Figure 5: (top) C-Bracket in Eco-Car, and (bottom) mass of the original structure

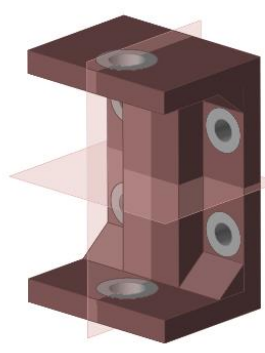

Figure 6: Design Space and Non-Design

Non-design spaces are defined separately to ensure that critical mounting elements, such as bushing housings and bolt holes, are not affected by the optimization process design - red regions in Figure 6 indicate design space and grey regions are non-design spaces. 
Loads and constraints were then applied from existing known load cases and adapted for the bracket. Six envisaged worst-case load combinations were considered to guarantee the bracket's safety under extreme circumstances, these being combined into an overall envelope, enhancing the safety margin to a hyper-conservative degree. Symmetry constraints about two planes were imposed to ensure the final part can be orientated in any direction and on any side of the vehicle, as well as to simplify the computational overhead in analysis.

TO analyses were carried out for over 60 iterations, using various optimization constraints to generate an optimal part. As more extreme target volumes were explored, manufacturing constraints began to dictate limits of the optimization, so a minimum dimension was imposed to avoid too small a cross-section being generated to be viably printed in the AM process.

Table 1 below lists the 10 best iterations achieved by the optimization, based on mass, while still achieving a safe stress limit - here target volume is the input, and Factor of Safety, Total Mass, max. Major Principal Stress, max. von Mises Stress and max. Displacement the analysis outputs of the TO process. The tensile strength of Ti64 is 1000 [MPa].

\begin{tabular}{|c|c|c|c|c|c|c|c|}
\hline $\begin{array}{c}\text { Target } \\
\text { Volume }\end{array}$ & $\begin{array}{c}\text { Iteration } \\
\text { No. }\end{array}$ & Load Case & $\begin{array}{c}\text { Minimum } \\
\text { Factor of } \\
\text { safety }\end{array}$ & $\begin{array}{c}\text { Total } \\
\text { Mass } \\
{[\mathrm{g}]}\end{array}$ & $\begin{array}{c}\text { Maximum Major } \\
\text { Principal Stress } \\
{[\mathrm{MPa}]}\end{array}$ & $\begin{array}{c}\text { Maximum von } \\
\text { Mises Stress } \\
{[\mathrm{MPa}]}\end{array}$ & $\begin{array}{c}\text { Maximum } \\
\text { Displacement } \\
{[\mathrm{mm}]}\end{array}$ \\
\hline $4 \%$ & 45 & load case envelope & 2.4 & 24 & 369 & 347 & 0.676 \\
\hline $7 \%$ & 27 & load case envelope & 3.9 & 28 & 304 & 213 & 0.438 \\
\hline $8 \%$ & 26 & load case envelope & 4.9 & 30.3 & 233 & 168 & 0.368 \\
\hline $10 \%$ & 25 & load case envelope & 6.2 & 34.6 & 182 & 133 & 0.297 \\
\hline $11 \%$ & 24 & load case envelope & 6.6 & 36.7 & 170 & 126 & 0.275 \\
\hline $13 \%$ & 23 & load case envelope & 5.5 & 40.3 & 162 & 115 & 0.246 \\
\hline $15 \%$ & 18 & load case envelope & 7.9 & 44.8 & 142 & 104 & 0.215 \\
\hline $20 \%$ & 17 & load case envelope & 9.9 & 55.7 & 110 & 83.3 \\
\hline $25 \%$ & 16 & load case envelope & 11.7 & 68.3 & 94.8 & 70.8 & 0.178 \\
\hline $30 \%$ & 15 & load case envelope & 13.6 & 81.2 & 84.8 & 6.156 \\
\hline
\end{tabular}

Table 1: Topology optimization iterations in order of ascending mass

As more aggressive target volumes were tested, there was a corresponding increase in Major Principal, von Mises stresses as well as Displacement. This was expected as the bracket has numerous constraints in the optimization process, such as symmetry about two planes as shown in Figure 6 and a low overall volume relative to the loading involved; these constraints stop the topology optimization process from producing branch-like structures which tend to, as target volume decreases, create non-linear jumps in stresses at branch events.

Also shown in Table 1 above, is that as the mass of an optimized part decreases, the maximum displacement increases. This shows the sacrifice of stiffness to achieve lighter components shared by most topology optimizations with the goal of smaller volumes. 
The most aggressive target volume achieved was $4 \%$ of the original design volume with a mass of $24[\mathrm{~g}]$ and an overall minimum safety factor of 2.4 , this mainly due to geometric and constraint effects.
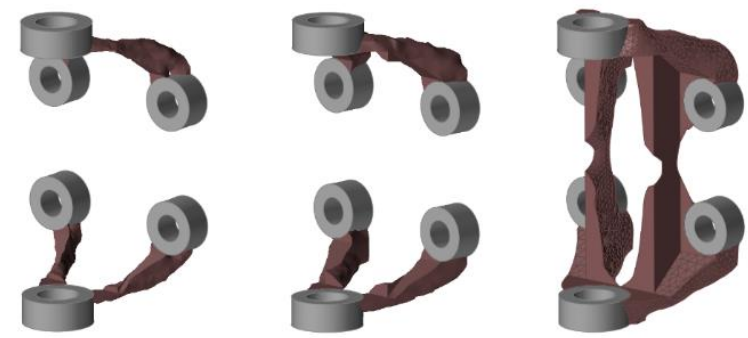

Figure 7: varying target volumes produced by TO analysis iterations

As the target volume goal decreased (Figure 7), the two halves of the bracket begin to separate, creating a less stiff part while decreasing the weight - this would also result in significant misalignment challenges on installation, affecting performance.

The next step was to generate a smooth free-form body using PolyNURBS techniques (Figure 8), thereby eliminating all the jagged/tessellated features resultant from the topology optimization process.

A design decision was made to join the two symmetrical halves with a bridge of material ensuring ease of alignment during installation and to further stiffen the structure. Finally, geometric entities were created on any significant edges to further reduce any sharpness to improve printability overall - the whole iterative analysis procedure taking approximately 180 hours in total for the full process.

\subsubsection{Design Evolution \& Simulation Results}

Figure 9 below depicts the evolvement of the optimized design in Ti6-4 showing projected weight-saving of $41 \%$ on this component alone, from the original carbon-fibre bracket on the vehicle to final

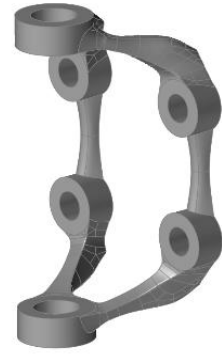

Figure 8: smoothed TO part generated utilizing PolyNURBS computational model form.
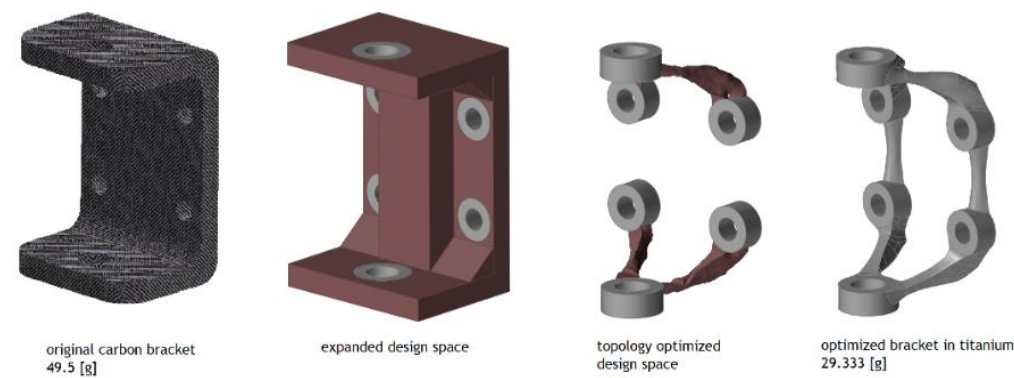

Figure 9: design evolution of C-Bracket from carbon to optimized Ti-6-4 AM design

After smoothing via PolyNURBS, further FEA tests were carried out on the final structure to verify no initial design constraints were unintentionally violated, resulting in expected improved results than from the raw, unrefined structure resultants in the TO iterations - as all stress concentrations from any tessellated surfaces would have been relieved, and additional material would have also been added to the design as a result - Figure 10 depicts samples of these results showing Displacement, von Mises and Major Principal stress outputs. 


\subsubsection{Final Product}

The final design model was sent through for additive manufacturing in Ti64 at various facilities in a round robin study - which will be reported elsewhere - and non-destructive tests were conducted post-printing to establish conformity of the final component with the design.
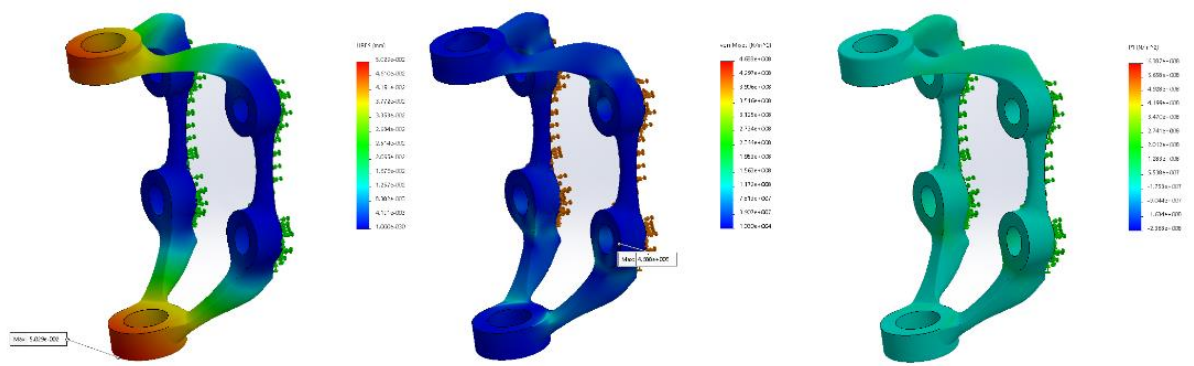

Figure 10: finite element analysis on smoothed component depicting:

(l) resultant displacement, (c) von Mises stress, and ( $r$ ) major principal stress

Parts in this paper were manufactured using laser powder bed fusion using standard process parameters in an EOS M280 system at Central University of Technology. Typical parts from this process have been analyzed in detail previously (Yadroitsev et al[20]; du Plessis et al[21]).

Typical parts from this process contain minimal porosity, and $10 \mathrm{~mm}$ test cubes were also printed with the first C-Bracket as comparative test specimens - these were then analyzed at high resolution to confirm low levels of porosity; the use of microCT in AM (du Plessiset al[8]) is globally an accepted industry standard. MicroCT was performed at the Stellenbosch CT Scanning facility (du Plessis et al[22]). The cube volume was measured using accurate sub-voxel surface determination in microCT data, and combined with mass from an accurate scale, provided a mean density of 4.504 $\left[\mathrm{g} / \mathrm{cm}^{3}\right]$, well within the Ti6Al4V density range.

The microCT images (Figure 11) show some small porosity as expected with a total volumetric percentage of $0.012 \%$. They depict the microCT data of this $10 \mathrm{~mm}$ cube as a surface- and transparent-view showing the even distribution of the micro-porosity. Larger parts, difficult to test at this resolution by microCT, are expected to have the same microporosity distribution, considered acceptable at this low level, as long as it is evenly distributed.
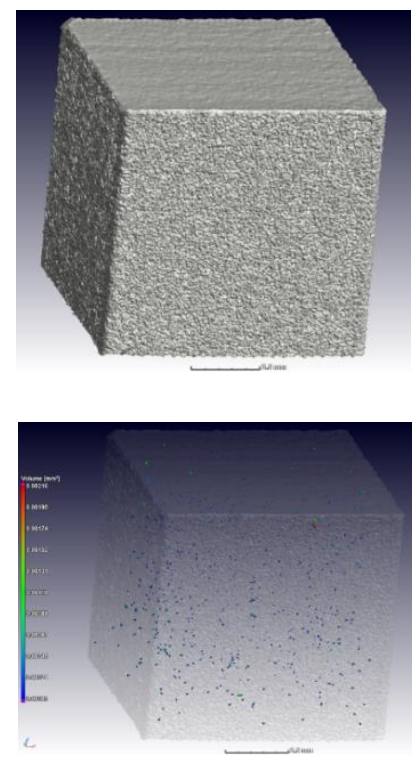

Figure 11: Solid $10 \mathrm{~mm}$ cube subjected to high-res microCT (l) shows surface and (r) shows micro-porosity distribution and colour coding by volume. 
The final topology optimized Ti-6-4 product (Figure 12) is shown after stress-relief heat treatment, as well as removal of support material \& base support; no further physical post-processing was carried out. Also depicted are standard pieces utilized in the testing process for detailed analysis of mean density, porosity, and possible stop-start flaws.

The brackets were then tested via microCT, and the microCT scan on the C-bracket (compared to the $10 \mathrm{~mm}$ cube) showed no macro-porosity. Large unexpected pores or flaws can sometimes be present due to major build errors or contamination, for example. They were found to have no detectable porosity $>135 \mu \mathrm{m}$ or unexpected internal flaws either. Compared to the CAD, the two arms of the bracket slightly warped inwards by a maximum of $0.5 \mathrm{~mm}$ on both sides, with horizontal offset also approx. $0.5 \mathrm{~mm}$ (Figure 13).

The mass of each Ti-6-4 metal printed C-bracket is 27.0 grams saving $45 \%$ compared to the original design, which was an already very light carbon-fibre part. The mass progression (Table 2 below) of the C-Bracket structure through the whole process was as follows:

\begin{tabular}{|l|c|}
\hline \multicolumn{1}{|c|}{ structure } & mass [g] \\
\hline original carbon structure with inserts & 49.5 \\
\hline structure reverted to solid titanium & 177.29 \\
\hline titanium structure topology optimized & 24 \\
\hline polyNURBed smoothed solution & 29.33 \\
\hline final printed part & 27 \\
\hline
\end{tabular}

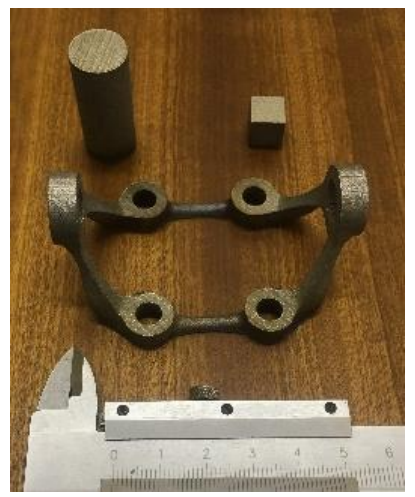

Table 2, mass progression of C-Bracket design
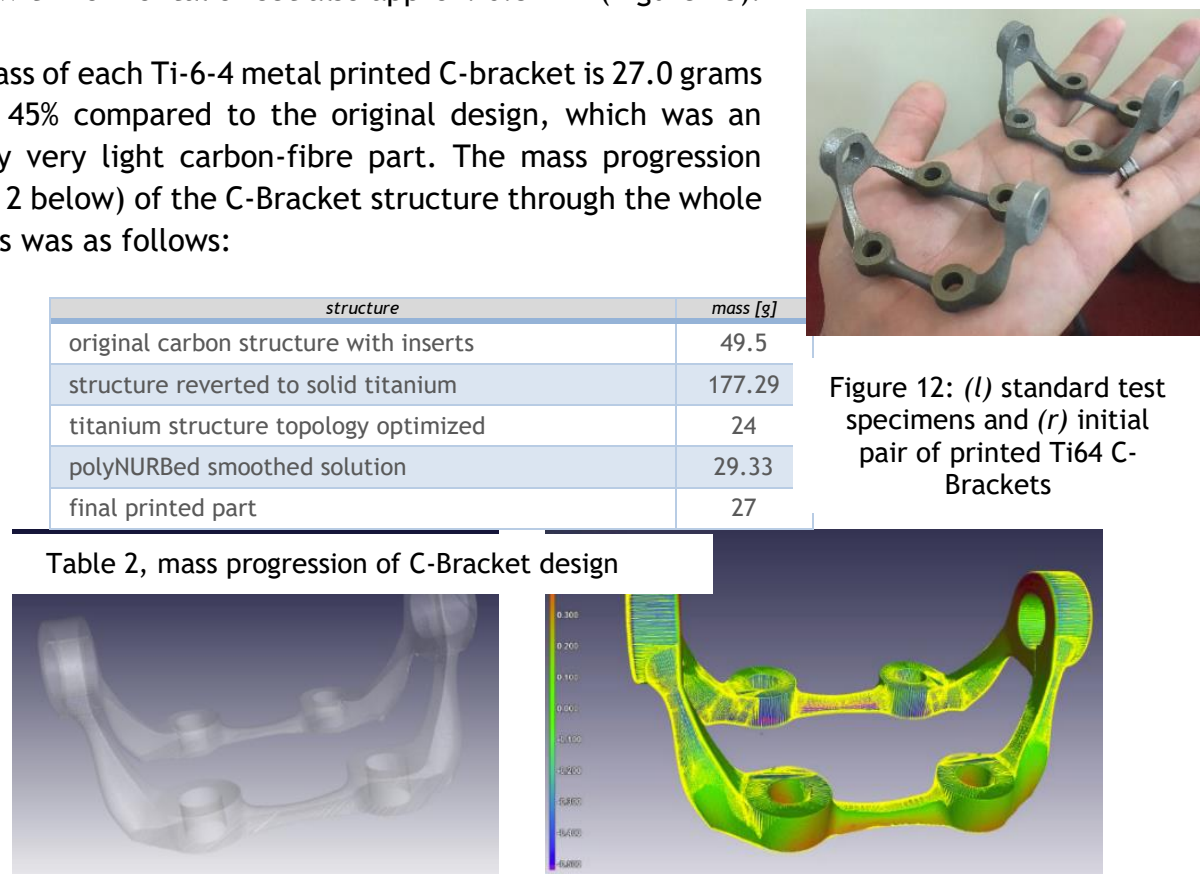

Figure 12: (l) standard test specimens and $(r)$ initial pair of printed Ti64 CBrackets

Figure 13: the microCT result showing (l) no internal porosity, and (r) CAD variance of printed model to original CAD model

\subsection{Case Study 2: Steering Arch Design Process}

\subsubsection{Initial Design Need}

The existing steering arch is a carbon structure, designed to neatly surround the driver's legs (Figure 14). The existing structure was cut from high-density polystyrene block via robot-CNC and the structure then over-laid with multiple layers of carbon-fibre.

This structure has proved itself more than up to the task, stiffness and compliance-wise, over several competitions already, so this choice was mainly based on three issues:

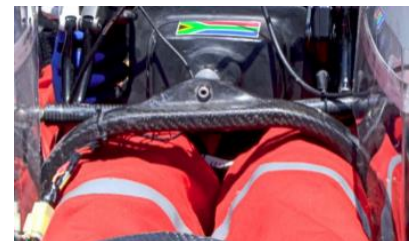

Figure 14: Original Steering Arch

- increasing stiffness of the structure to improve loss of efficiency through the steering, 
- to consolidate parts to one single component, thereby saving weight,

- to explore how additional latticed optimization could further enhance the design

To further attempt to add to the general light-weighting requirement and efficiency of design, it was decided very early in the design process to apply a lattice-work design optimization to the structure. The deployment of a latticed Ti-6-4 structure will appreciably add to the stiffness of the component, which is important as it carries both front corners as well as the steering system \& all driver controls, and any compliance that may exist there.

\subsubsection{Design \& Optimization Process}

Again, existing geometry in CAD was checked to ensure conformity to the physical component. All loading and constraints were then adapted to the new consolidated design space. The Design Space (Figure 15) expanded as far as practicable to allow the topology optimization free-reign to generate alternative un-envisaged options.

Non-Design and Design Spaces were then defined to protect vital connection volumes from being edited by the optimization. Topology Optimization is then first applied and finalized before the lattice optimization is carried out.

The goal of the initial TO studies was to provide a stiffenough base model for the subsequent lattice optimization to further reduce material, ensuring the final latticed structure has enough stiffness to withstand a chosen max. deflection of $1.0[\mathrm{~mm}]$, while also allowing it to still lightweight the design. At this stage too, an imposition of overhang angle of $40^{\circ}$ was imposed to ensure min. support

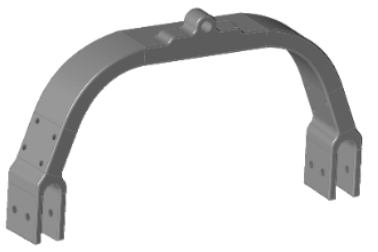

Figure 15: Expansion of design space from the (above) original design, and (below) the expanded Design Space with Non-Design spaces

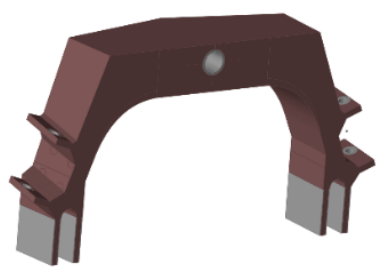
material in the print would be created. This would also dictate the lattice structure formation in that phase of the analysis.

Table 3 below shows 5 selected iterations of the topology optimization phase out of 65 carried out, with the objective of maximizing stiffness while removing enough weight for the lattice optimization to still be competitive. The inputs were Target Volume and Maximize Stiffness while the outputs were Minimum Factor of Safety, Total Mass, Maximum Major Principle Stress, Maximum von Mises Stress and Maximum Displacement.

\begin{tabular}{|c|c|c|c|c|c|c|c|}
\hline $\begin{array}{c}\text { Target } \\
\text { Volume }\end{array}$ & $\begin{array}{c}\text { Iteration } \\
\text { No }\end{array}$ & Load Case & $\begin{array}{c}\text { Minimum } \\
\text { Factor of } \\
\text { safety }\end{array}$ & $\begin{array}{c}\text { Total Mass } \\
{[\mathrm{kg}]}\end{array}$ & $\begin{array}{c}\text { Maximum } \\
\text { Major } \\
\text { Principal } \\
\text { Stress } \\
{[\mathrm{MPa}}\end{array}$ & $\begin{array}{c}\text { Maximum } \\
\text { von Mises } \\
\text { Stress [MPa] }\end{array}$ & $\begin{array}{c}\text { Maximum } \\
\text { Displacement } \\
{[\mathrm{mm}]}\end{array}$ \\
\hline $\mathbf{2 0 \%}$ & 29 & load case envelope & 11 & 1.63 & 68.7 & 75.4 & 0.118 \\
\hline $\mathbf{1 5 \%}$ & 48 & load case envelope & 14.4 & 1.36 & 70.5 & 57.3 & 0.203 \\
\hline $10 \%$ & 46 & load case envelope & 15.1 & 1.10 & 67.6 & 54.8 & 0.241 \\
\hline $\mathbf{8 \%}$ & 50 & load case envelope & 8 & 0.986 & 88.8 & 103 & 0.270 \\
\hline $\mathbf{5 \%}$ & 44 & load case envelope & 5.3 & 1.09 & 166 & 155 & 0.317 \\
\hline
\end{tabular}

Table 3: selected TO iterations to obtain optimal design volume in order of ascending displacement

As the volume of the arch is decreased the maximum stresses present do not always decrease, this is due to varying branching structures developed by the optimization sacrificing strength for stiffness. Iteration 29 achieved the lowest maximum displacement while remaining in range of the final mass target for the lattice stage. It also was the only iteration to produce more than one arch across the gap which increases the stiffness at the lattice stage output. 
PolyNURBS techniques were used to smooth out any jagged/tesselated edges produced by the TO analyses, thereby preparing the model for a further lattice optimization phase. The primary constraint to this was a pre-advised manufacturing constraint of a $2[\mathrm{~mm}] \mathrm{min}$. diameter for any lattice beams in the component. This substantially limited the min. mass achievable as smaller lattice structures, particularly micro lattice structures, would have performed better on a weight saving basis, whilst still retaining design objectives.

Lattice optimizations were then performed varying input parameters and constraints to reduce the weight of the final arch design. Table 4 below shows the 10 best iterations out of a total of 89 carried out, in order of ascending mass, with the input parameters of target length, minimum diameter and maximum diameter of lattice beam sections, while the outputs are FEA results such as minimum factor of safety, maximum major principle stress, maximum von Mises stress and maximum displacement as well as the mass of the component.

\begin{tabular}{|c|c|c|c|c|c|c|c|}
\hline $\begin{array}{l}\text { Lattice Constraints } \\
\text { (Target Length/ } \\
\text { Min. Diam/ } \\
\text { Max. Diam) [mm] }\end{array}$ & Iteration & Load Case & $\begin{array}{l}\text { Minimum } \\
\text { Factor of } \\
\text { safety }\end{array}$ & $\begin{array}{l}\text { Total } \\
\text { Mass } \\
{[\mathrm{kg}]}\end{array}$ & $\begin{array}{c}\text { Maximum } \\
\text { Major } \\
\text { Principal } \\
\text { Stress } \\
\text { [MPa] }\end{array}$ & $\begin{array}{l}\text { Maximum von } \\
\text { Mises Stress } \\
\text { [MPa] }\end{array}$ & $\begin{array}{l}\text { Maximum } \\
\text { Displacement } \\
{[\mathrm{mm}]}\end{array}$ \\
\hline $21-2-2$ & 61 & load case envelope & 2.8 & 752 & 58.6 & 301 & 1.41 \\
\hline $20-2-2$ & 60 & load case envelope & 1.4 & 762 & 150 & 591 & 1.24 \\
\hline $19-2-2$ & 59 & load case envelope & 4.5 & 772 & 61.5 & 150 & 1.17 \\
\hline $18-2-2$ & 58 & load case envelope & 3.5 & 777 & 86 & 235 & 1.13 \\
\hline $21-2-2.1$ & 75 & load case envelope & 3.2 & 799 & 58.4 & 257 & 1.30 \\
\hline $19-2-5$ & 34 & load case envelope & 5.2 & 800 & 81.6 & 151 & 0.98 \\
\hline $19-2-4$ & 52 & load case envelope & 5.2 & 800 & 79.1 & 140 & 0.978 \\
\hline $19-2-3$ & 69 & load case envelope & 5.1 & 801 & 74 & 111 & 0.977 \\
\hline $17-2-2$ & 65 & load case envelope & 3.3 & 813 & 76.7 & 250 & 1.01 \\
\hline $20-2-5$ & 35 & load case envelope & 3.9 & 815 & 147 & 214 & 0.952 \\
\hline
\end{tabular}

Table 4: selected topology optimization iterations to obtain optimal design volume in order of ascending displacement

Table 4 shows, as lattice beams become ever more slender, greater mass savings can obviously be achieved, but that stresses do not tend to converge to their limits in relation to mass as usually seen in optimizations - this was due to stress concentrations being relocated to different points on the lattice structure, and each mesh size having different optimal diameter inputs, restricted by the $2[\mathrm{~mm}] \mathrm{min}$. diameter print machine hardware constraint.

Also, the max. displacement increases by as much as 14 -fold over the purely TO component, showing how lattice structures tend to deflect while still maintaining relative strength and reducing mass. This, however, was still well within the imposed design constraint.

The lowest mass of 752 [g] was achieved by iteration 61 with a min. safety factor of 2.8; this safety factor caused by stress concentrations that were resolved and improved on in the following smoothing phase. This particular iteration also created some slender lattice beams and voids in some areas, which would be unprintable by available printers. However, a subsequent smoothing phase would remedy all of these anomalies.

The next major challenge was to ensure that there was clear delineation between the lattice structures and the support material generated by the printing process, and to eliminate any possibility of the lattice being compromised in post-processing removal of support material.

Support shell surfaces in a range of 1-2 [mm] thickness were then created to facilitate this, taking into consideration the optimum orientation on the printer bed. Furthermore, the overall outer design extents of the steering arch was such that it excluded the possibility of being printed on any of the available printer options in the country, bar one at AeroSwift, and this itself has constraints that dictated the orientation of the component, and hence the amount of support material created, and its connection points to the printed structure. 
Horizontal ('face-down' - Figure 16) or vertical ('upright' - Figure 17) orientations proved to be the best options, showing the support shell surfaces that would need to be created to facilitate this, as well as the orientation on the available print-beds.
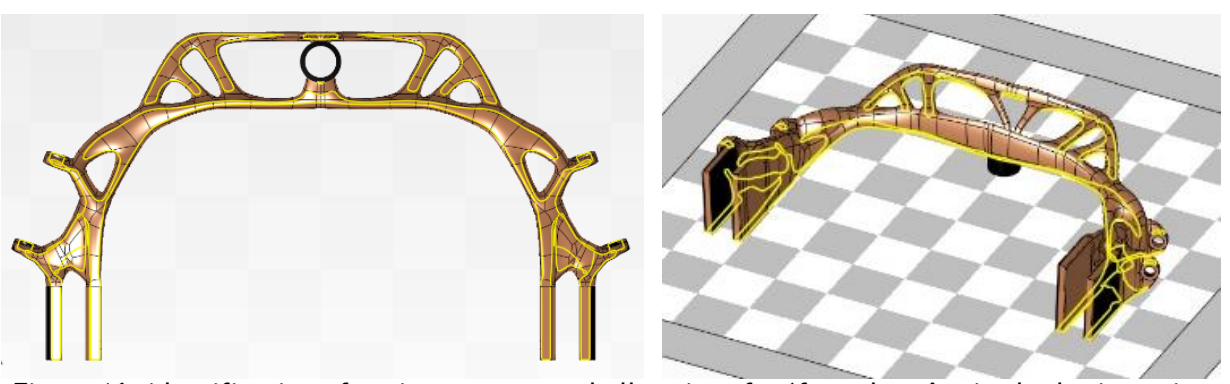

Figure 16: identification of optimum support shell regions for 'face-down' print-bed orientation (l) front view depicting required shell regions, and $(r)$ the structure orientated on the print bed
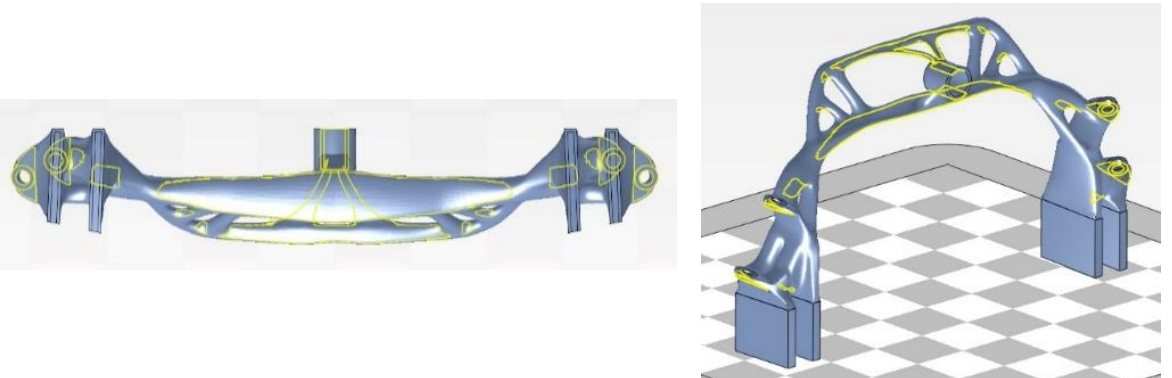

Figure 17: identification of optimum support shell regions for 'upright' print-bed orientation

(l) bottom view depicting required shell regions, and $(r)$ the structure orientated on the print bed

This was a major challenge due to restricted capability on current available software and was not easily achievable, requiring jumping between multiple software platforms to achieve successful integration. These surfaces needed to be placed between the lattice beams and the support material structures, yet still ensuring direct connection. This is easily achievable in some high-end platforms, but, even in academic option, they are financially prohibitive.

As depicted in Figure 18 below, the optimal generated support material, in blue, joining to the components defined support material shell structures is displayed for both orientations this was the most efficient layout iro support material joining onto the main arch structure, as well as available print bed constraints, according to available software options.
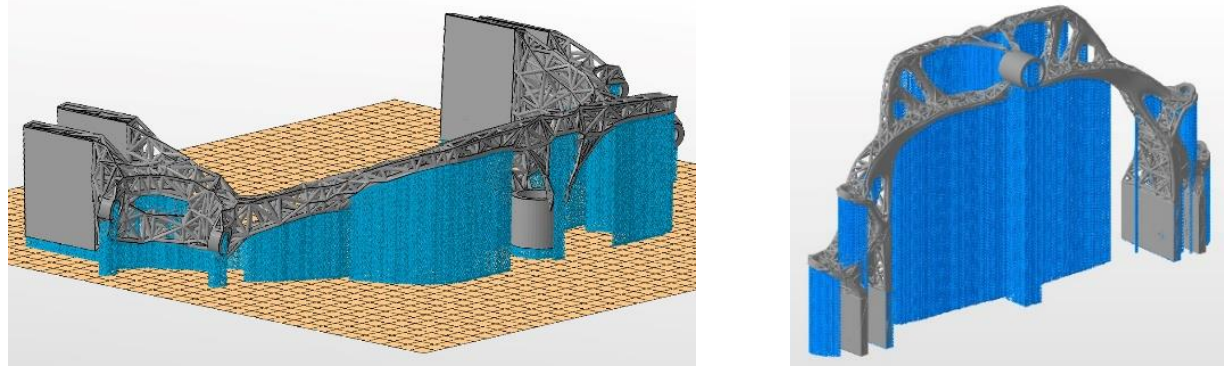

Figure 18: support material generation based on print-bed orientation

(l) face-down orientation, and ( $r$ ) upright orientation

In a unique application of microCT-based voxel software, the component was further smoothed using image processing methods (Figure 19) with the yellow envelope line showing a smoothed model compared to the original design (white). This removes sharp corners and ridges, which may act as stress raisers and could also make final printing challenging. 

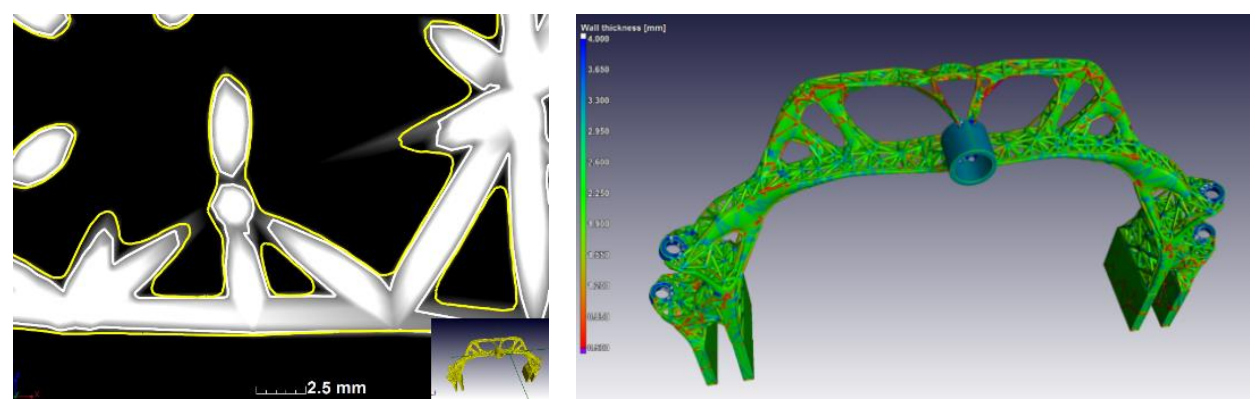

Figure 19: (l) Voxel-based image smoothing of the final model (yellow lines), and (r) the final model wall thickness analysis, to check the final design prior to printing

The final smoothed part is then subjected to Wall Thickness Analysis to ensure no parts are too thin. Despite minimal strut thickness constraints, some struts may be created thinner. When they are non-circular in cross-sectional shape, the smoothing process may make them significantly thinner than expected. This process of smoothing (Figure 20) may be modified if this is the case. Final model volume can be checked as this process fills in significant amounts of material up to about $30 \%$ volume increase, increasing the weight of the arch to 857 [g].
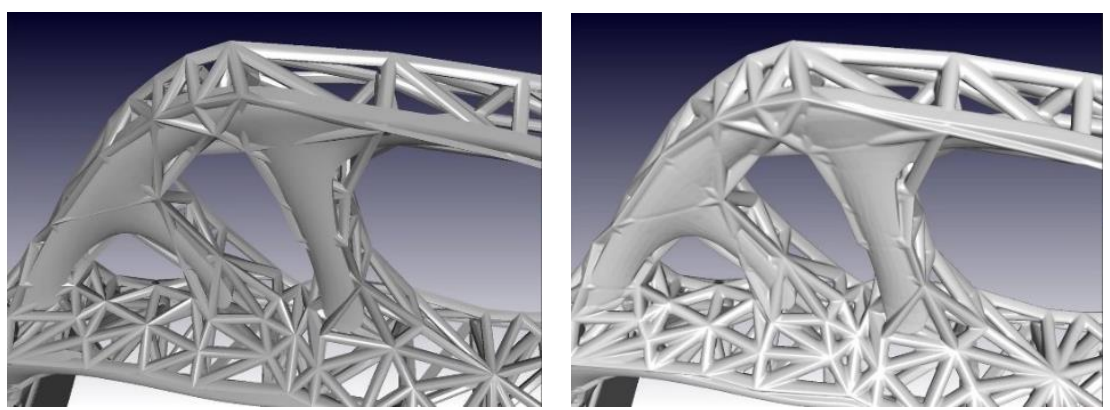

Figure 20: close-up comparison between (l) original model STL file, and $(r)$ the final voxel-based smoothed part

The final smoothed latticed structures including support material shell surfaces are depicted in Figure 21 below. The full iterative analysis procedure taking approx. 500 hours.
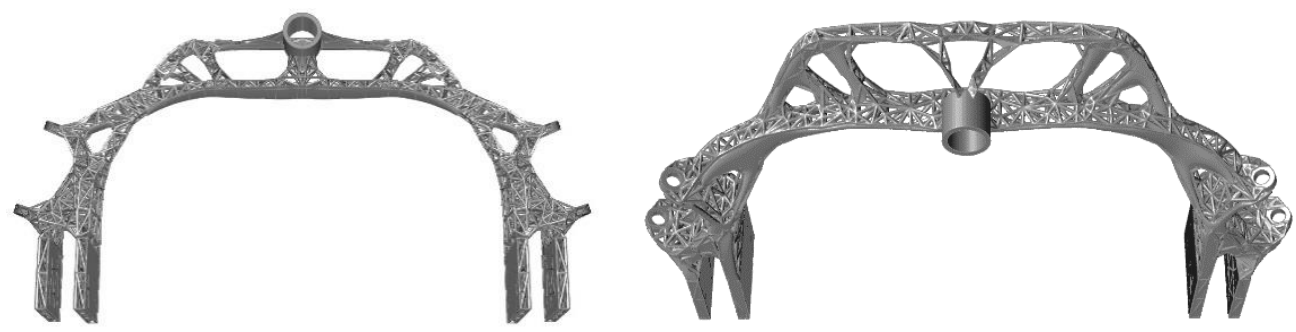

Figure 21: Smoothed Topology Optimized and Latticed steering arch, including solid 'shell' sections for support material connection (l) for upright orientation, and (b) for face-down

\subsection{Design Evolution \& Simulation Results}

Figure 22 shows the design evolvement of the optimized, latticed design in Ti-6-4 with a projected mass-saving of $14 \%$, from the original carbon-fibre steering arch on the vehicle. 

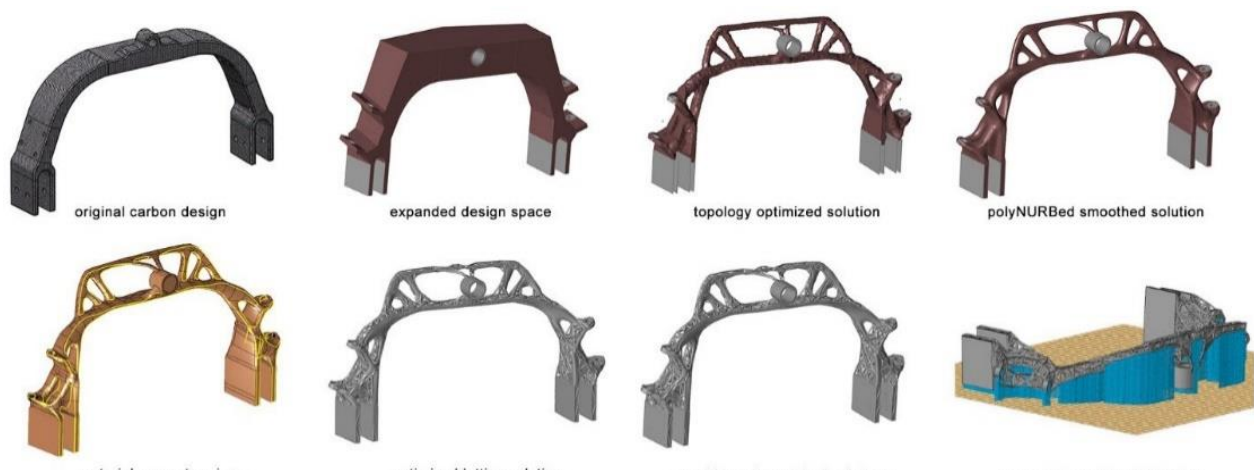

voxel-based smoothed solutuon

support material optimization

Figure 22: design evolution of Steering Arch from carbon to optimized \& latticed Ti-6-4 AM design for face-down print

After smoothing via PolyNURBS, further FEA tests were carried out on the final computational model to verify that initial design constraints were not unintentionally violated, resulting in expected improved results than from the raw, unrefined structure resultants from the TO iterations depicted in Table 4 - Figure 23 depicts a sample of some of these results showing Displacement and von Mises stress: the critical stress zones being understandably where the lattice meets the shelled monocoque insertion regions - however, this is still well within the design constraints, and would also be improved by the voxel-based smoothing process.
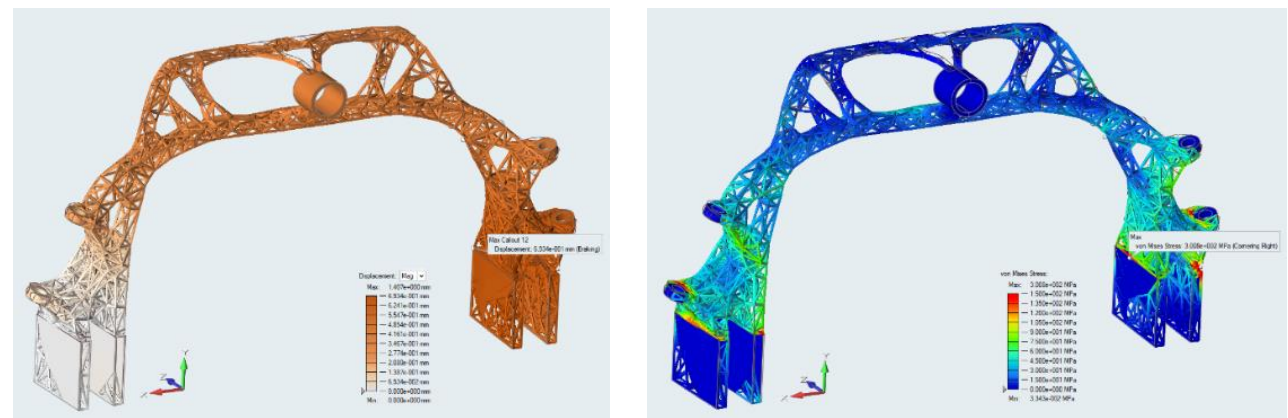

Figure 23: selected finite element analysis solutions on smoothed polyNURBed component depicting: (l) resultant displacement, and ( $r$ ) von Mises stress

\subsubsection{Final Product}

At the point of submission of this paper, the printed final product was still due for printing at AeroSwift, a government-backed project launched in collaboration between Aerosud and the CSIR. As it turned out, the printer orientation would be dictateded by printer constraints, and the upright orientation would be the only option available due to print-bed restrictions, necessitating further adjustments to the design to accommodate the configuration - further compliance, porosity and variance testing will be carried before installation into the Eco-Car.

The projected mass of the Ti-6-4 metal printed Steering Arch is 857.0 [g] saving $16 \%$ compared to the original design, which was an already very light carbon-fibre part. The mass progression (Table 5 below) through the whole process was as follows:

\begin{tabular}{|l|c|}
\hline \multicolumn{1}{|c|}{ structure } & mass [g] \\
\hline original carbon structure with inserts & 994.16 \\
\hline structure reverted to solid titanium & 2805 \\
\hline titanium structure topology optimized & 1630 \\
\hline polyNURBed solution & 1840 \\
\hline latticed structure & 752 \\
\hline
\end{tabular}


smoothed lattice structure 857

Table 5, mass progression of C-Bracket design

\section{CONCLUSIONS}

In this work, two case studies of topology optimized and latticed optimized components were presented. These case studies demonstrated the topology optimization process, with its challenges and practical aspects. The bracket was produced successfully and the use of microCT showed lack of major porosity and acceptable variance between computational and physical product. This process demonstrated how AM techniques can be used to further lightweight components from an already-lightweight composite option in an experimental vehicle.

The second case study (that of a steering arch) incorporated lattice optimization into a topology optimized design. The design process was outlined, especially the addition of a solid shell structure to simplify the removal of support material from the part, and also highlighted challenges encountered in a process of this nature with both current hardware and software.

A unique final smoothing phase using voxel-based image analysis software was also demonstrated including wall thickness analysis validation utilizing the same software. The steering arch is now ready for production on a large-bed additive manufacturing system, to be tested in the NMU Eco-Car, and which will be reported in future work.

\section{REFERENCES}

[1] Yang, Sheng, \& Yaoyao, and Fiona Zhao. n.d. "Additive Manufacturing-Enabled Design Theory and Methodology: A Critical Review." Accessed June 15, 2018. https://doi.org/10.1007/s00170-015-6994-5.

[2] du Plessis, A., I. Yadroitsava, I. Yadroitsev, S. G. le Roux, and D. C. Blaine. "Numerical comparison of lattice unit cell designs for medical implants by additive manufacturing." Virtual and Physical Prototyping (2018): 1-16.

[3] Zhang, Xiang-Yu, Gang Fang, and Jie Zhou. 2017. "Additively Manufactured Scaffolds for Bone Tissue Engineering and the Prediction of Their Mechanical Behavior: A Review." Materials 10 (1). Multidisciplinary Digital Publishing Institute: 50. https://doi.org/10.3390/ma10010050.

[4] Wang, Xiaojian, Shanqing Xu, Shiwei Zhou, Wei Xu, Martin Leary, Peter Choong, M Qian, Milan Brandt, and Yi Min Xie. 2016. "Topological Design and Additive Manufacturing of Porous Metals for Bone Scaffolds and Orthopaedic Implants: A Review." Biomaterials 83 (March): 127-41. https://doi.org/10.1016/j.biomaterials.2016.01.012.

[5] Orme, Melissa E., Michael Gschweitl, Michael Ferrari, Ivan Madera, and Franck Mouriaux. 2017. "Designing for Additive Manufacturing: Lightweighting Through Topology Optimization Enables Lunar Spacecraft." Journal of Mechanical Design 139 (10). American Society of Mechanical Engineers: 100905. https://doi.org/10.1115/1.4037304.

[6] du Plessis, A., C. Broeckhoven, I. Yadroitsev, I. Yadroitsava, and S.G. le Roux. 2018. "Analyzing Nature's Protective Design: The Glyptodont Body Armor." Journal of the $\begin{array}{llll}\text { Mechanical Behavior of Biomedical } & \end{array}$ https://doi.org/10.1016/j.jmbbm.2018.03.037.

[7] du Plessis, A., and Chris Broeckhoven. n.d. "METAL BODY ARMOUR: BIOMIMETIC ENGINEERING OF LATTICE STRUCTURES.” In RAPDASA 2018.

[8] du Plessis, A., Igor Yadroitsev, Ina Yadroitsava, and Stephan le Roux. 2018. "X-Ray Micro Computed Tomography in Additive Manufacturing: A Review of the Current Technology and Applications." 3D Printing and Additive Manufacturing In Press.

[9] Liu, Jikai, Andrew T. Gaynor, Shikui Chen, Zhan Kang, Krishnan Suresh, Akihiro Takezawa, Lei $\mathrm{Li}$, et al. 2018. "Current and Future Trends in Topology Optimization for Additive Manufacturing." Structural and Multidisciplinary Optimization, May. Springer Berlin Heidelberg, 1-27. https://doi.org/10.1007/s00158-018-1994-3

[10] Robot Bike Company. 2016. Mountain Bicycles and Additive Manufacturing (customer story). [online]. Retrieved from: https://www.solidthinking.com/resources/all-products/customerstories/. 
[11] Ryerson University. 2016. Ryerson's International Hyperloop Team (customer story). [online]. Retrieved from: https://www.solidthinking.com/resources/all-products/customer-stories/.

[12] Gator Motorsports. 2017. Automotive/Formula Racing (customer story). [online]. Retrieved from: https://www.solidthinking.com/resources/all-products/customer-stories/

[13] Zhu, JH., Zhang, WH. \& Xia, L. 2016. Topology Optimization in Aircraft and Aerospace Structures Design, Archive of Computational Methods in Engineering, 23(1), pp 595-622.

[14] Yildiz, AR. 2008. Optimal Structural Design of Vehicle Components Using Topology Design and Optimization, Materials Testing, 50(4) , pp 224-228.

[15] Vatanabe, SL., Lippi, TN., de Lima, CR., Paulino, GH., Silva, CN. 2016. Topology optimization with manufacturing constraints: A unified projection-based approach, Advances in Engineering Software, 100(1), pp 97-112.

[16] Gibson, I., Rosen, D., Stucker, B. 2015. Additive Manufacturing Technologies, 2nd Edition, Springer-Verlag New York.

[17] Optistruct User Guide. 2017. [online]. Available: https://connect.altair.com/CP/kbview.html?f=2\&kb=167280.

[18] Gaynor, AT., Guest, JK. 2016. Topology optimization considering overhang constraints: Eliminating sacrificial support material in additive manufacturing through design, Structural and Multidisciplinary Optimization, 54(5), pp 1157-1172.

[19] Helou, M. \& Kara, S. 2017. Design, analysis and manufacturing of lattice structures: an overview, International Journal of Computer Integrated Manufacturing, 31(3), pp 243-261.

[20] Yadroitsev, I., P. Krakhmalev, I. Yadroitsava, and A. du Plessis. 2018. "Qualification of Ti6Al4V ELI Alloy Produced by Laser Powder Bed Fusion for Biomedical Applications." JOM. https://doi.org/10.1007/s11837-017-2655-5.

[21] du Plessis, A., Stephan Gerhard le Roux, Gerrie Booysen, and Johan Els. 2016. “Quality Control of a Laser Additive Manufactured Medical Implant by X-Ray Tomography." 3D Printing and Additive Manufacturing. https://doi.org/10.1089/3dp.2016.0012.

[22] du Plessis, A., S.G. le Roux, and A. Guelpa. 2016. "The CT Scanner Facility at Stellenbosch University: An Open Access X-Ray Computed Tomography Laboratory." Nuclear Instruments and Methods in Physics Research, Section B: Beam Interactions with Materials and Atoms 384. https://doi.org/10.1016/j.nimb.2016.08.005. 